Journal of
Translational Medicine

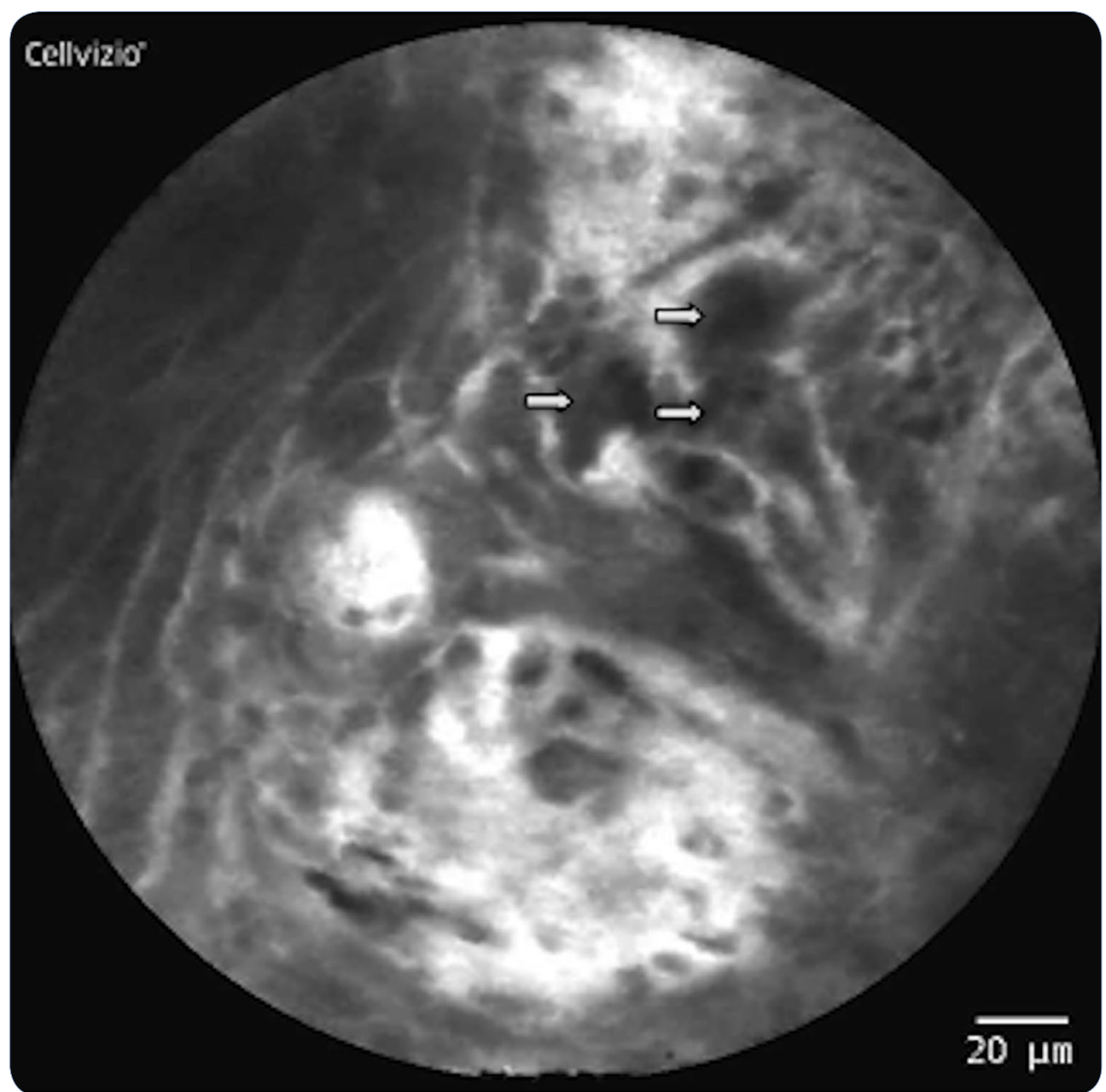

Development and validation of a classification and scoring system for the diagnosis of oral squamous cell carcinomas through confocal laser endomicroscopy

Oetter et al.

() Biomed Central 


\title{
Development and validation of a classification and scoring system for the diagnosis of oral squamous cell carcinomas through confocal laser endomicroscopy
}

Nicolai Oetter ${ }^{1 *}$, Christian Knipfer ${ }^{1,6}$, Maximilian Rohde ${ }^{1,6}$, Cornelius von Wilmowsky ${ }^{1}$, Andreas Maier ${ }^{2,6}$, Kathrin Brunner ${ }^{3}$, Werner Adler ${ }^{4}$, Friedrich-Wilhelm Neukam ${ }^{1,6}$, Helmut Neumann ${ }^{5,6 \dagger}$ and Florian Stelzle ${ }^{1,6+}$

\begin{abstract}
Background: Confocal laser endomicroscopy (CLE) is an optical biopsy method allowing in vivo microscopic imaging at 1000-fold magnification. It was the aim to evaluate CLE in the human oral cavity for the differentiation of physiological/carcinomatous mucosa and to establish and validate, for the first time, a scoring system to facilitate CLE assessment.

Methods: The study consisted of 4 phases: (1) CLE-imaging (in vivo) was performed after the intravenous injection of fluorescein in patients with histologically confirmed carcinomatous oral mucosa; (2) CLE-experts $(n=3)$ verified the applicability of CLE in the oral cavity for the differentiation between physiological and cancerous tissue compared to the gold standard of histopathological assessment; (3) based on specific patterns of tissue changes, CLE-experts $(n=3)$ developed a classification and scoring system (DOC-Score) to simplify the diagnosis of oral squamous cell carcinomas; (4) validation of the newly developed DOC-Score by non-CLE-experts $(n=3)$; final statistical evaluation of their classification performance (comparison to the results of CLE-experts and the histopathological analyses).

Results: Experts acquired and edited 45 sequences ( $260 \mathrm{~s}$ ) of physiological and 50 sequences ( $518 \mathrm{~s}$ ) of carcinomatous mucosa (total: 95 sequences/778 s). All sequences were evaluated independently by experts and non-experts (based on the newly proposed classification system). Sensitivity (0.953) and specificity (0.889) of the diagnoses by experts as well as sensitivity (0.973) and specificity (0.881) of the non-expert ratings correlated well with the results of the present gold standard of tissue histopathology. Experts had a positive predictive value (PPV) of 0.905 and a negative predictive value (NPV) of 0.945. Non-experts reached a PPV of 0.901 and a NPV of 0.967 with the help of the DOC-Score. Inter-rater reliability (Fleiss` kappa) was 0.73 for experts and 0.814 for non-experts. The intra-rater reliability (Cronbach's alpha) of the experts was 0.989 and 0.884 for non-experts.
\end{abstract}

Conclusions: CLE is a suitable and valid method for experts to diagnose oral cancer. Using the DOC-Score system, an accurate chair-side diagnosis of oral cancer is feasible with comparable results to the gold standard of histopathology - even in daily clinical practice for non-experienced raters.

Keywords: Confocal laser endomicroscopy, CLE, Oral squamous cell carcinoma, OSCC, Oral cancer, DOC-score, Real-time histology, Optical biopsy

\footnotetext{
*Correspondence: nicooetter@gmail.com

${ }^{\dagger}$ Helmut Neumann and Florian Stelzle contributed equally to this work

1 Department of Oral and Maxillofacial Surgery, Friedrich-Alexander

University Erlangen-Nürnberg (FAU), University Hospital Erlangen,

Glückstraße 11, 91054 Erlangen, Germany

Full list of author information is available at the end of the article
} 


\section{Background}

According to the GLOBOCAN series for the International Agency for Research on Cancer, there is an incidence of 14.1 million new cases of cancer worldwide per year. This number is expected to rise substantially over the next years [1]. Ten percent of all cancer cases are neoplasms of the head and neck area, of which more than $90 \%$ are classified as squamous cell carcinomas (HNSCCs) [2, 3]. Main risk factors for Squamous Cell Carcinomas (SCC) of the oral cavity are the consumption of tobacco or alcohol [4]. In recent times, HNSCCs with a Human Papilloma virus infection, especially HPV 16 , have been increasing [5] and represent about 20-25\% of all HNSCC cases [6]. Consequently, not only high-risk groups associated with alcohol and tobacco use but also a growing group of younger patients with HPV-infections are affected by cancer in the oral cavity.

Even though the therapy of HNSCCs has improved substantially-regarding the advancement in surgical methods and the development of specific radio and chemotherapy regimens over the last decades-one of the most crucial factors in the successful treatment of oral cancer still remains the early and accurate diagnosis [7-9].

The present gold standard for the diagnosis of oral malignant neoplasms starts with a visual gross identification of SCCs and its precursor lesions, followed by an invasive biopsy and a histopathological assessment [8]. The initial visual examination of the oral cavity is regarded as highly subjective, depends on the examiner's clinical experience and requires specific training $[5,10]$. The subsequent biopsy and histopathological tissue analysis are time and resource consuming and can also have individual variances in diagnosis $[11,12]$.

Optical imaging methods have the potential to fill the gap in daily clinical practice between the subjective visual examination and the invasive biopsy followed by a histopathological assessment. Confocal laser endomicroscopy (CLE) was recently introduced as a novel technique allowing in vivo microscopic evaluation of the tissue during the ongoing examination in real time by providing an optical biopsy $[3,13]$. This biomedical method could enable an in vivo diagnosis and can be reproduced almost indefinitely without iatrogenic damage to the patient and the need for additional human resources.

Recently, CLE has moved from a research tool to a valuable diagnostic technique in medical disciplines like gastroenterology [14-18]. Also preliminary results for a CLE diagnosis of oral cancer are promising [3, 13, 1926]. Transferring first experimental results of CLE in the human oral cavity into a valid and evidence based clinical setting is an essential step for this imaging tool on its way from bench to bedside [27].
The aim of our study was to depict the applicability of CLE examinations for diagnosing oral squamous cell carcinomas (OSCCs) in comparison to the gold standard of histopathological examination. Furthermore, we developed and evaluated, for the first time in this specialty and localization, an easy-to-use and straightforward scoring system for the identification of OSCCs on the basis of morphological and architectural criteria in order to enable a valid and reproducible CLE assessment even for non-experts in their daily clinical practice.

\section{Methods}

\section{Study design and setting}

An experimental clinical study was carried out to evaluate the clinical benefit of CLE assessment and furthermore to develop and validate a classification and scoring system for the diagnosis of squamous cell carcinoma of the oral cavity by using CLE with intravenous (IV) fluorescent dye. Prior to examinations, Institutional Review Board (IRB) approval was obtained (ethics committee of the University of Erlangen-Nürnberg; reference number: 243_12 B).

The study consisted of four phases: Phase 1, CLE Imaging. Phase 2, validation of the classification performance by experts $(\mathrm{n}=3)$ in differentiating between physiological and cancerous tissue with the help of CLE and comparison to histopathological analyses. Phase 3 , analysis of collected visual material and development of a classification and scoring system by CLE-experienced specialists. Phase 4, validation of the scoring system by non-experts in the field of CLE $(n=3)$ through assessment of the reliability in predicting the histopathological classification. Comparison of these results with the outcomes by experts and the gold standard of histopathology. Evaluation of the inter- and intra-rater reliability.

\section{Patients}

Our study is a survey of all inpatients of the Oral and Maxillofacial Surgery Department of the Erlangen University Hospital with histopathologically confirmed oral squamous cell carcinoma from 5/2013 to 7/2015. Exclusion criteria were defined as follows: age $<18$ years; current pregnancy/lactation period; allergies to fluorescein; renal insufficiency at any stage; beta-blocker medication. All lesions were histologically verified by the gold standard of biopsy and histopathological assessment by a pathologist specialized in cancer of the head and neck region, not involved in the CLE imaging.

\section{Examiners}

Two groups of independent examiners participated in the rating of CLE video sequences. The first group consisted of three medical experts with prior CLE experience. The 
second group (dentistry fellows; $\mathrm{n}=3$ ) served as nonexperts with no prior experience in CLE imaging.

\section{CLE-experimental setup and dye used}

Video sequences were recorded using a stand-alone probe-based CLE system (pCLE: ColoFlex UHD Probe, Cellvizio, Mauna Kea Technologies, Paris, France). This system offers a video frame rate of 12 frames/s, an image size of $576 \times 578$ pixels and a maximum penetration depth of $65 \mu \mathrm{m}$. The field of view has a diameter of $240 \mu \mathrm{m}$. Since normal tissue background fluorescence is not able to generate a sufficient signal-to-noise ratio [28], intravenous fluorescent dye was applied (fluorescein Alcon ${ }^{\circledR} 10 \%$ ). An initial dose of $3 \mathrm{~mL}$ prior to the examination with a maximum dose of $7.5 \mathrm{~mL}$ of fluorescein (IV) during the measurement procedure was defined according to experiences of our workgroup in the gastrointestinal tract.

\section{Procedure}

\section{Phase 1: CLE imaging}

All patients were informed about potential risks and signed an informed consent form prior to the study according to the IRB approval of the ethics committee of Friedrich-Alexander-University of Erlangen-Nürnberg (FAU, Approval Number 243_12 B). Examinations were performed on conscious patients and were totally integrated into the clinical procedure. To enhance image quality, the mouth was rinsed with acetylcysteine solution (ACC $100 \mathrm{mg}$ Hexal, effervescent tablets) by the patients prior to confocal imaging. After the fluorescent agent was injected, the probe was gently applied on the surface mucosa. In total, each patient had four sites imaged and every location was recorded as individual video sequences. Three reference points were determined for CLE imaging of physiological tissue: lower inner labium, upper alveolar ridge and palatal region. Last measured point was the lesion itself (OSCC). CLE imaging of the cancerous tissue was conducted in a standardized manner by focusing on the transition between physiological oral mucosa and the lesion with a subsequent adjustment on the center of the carcinoma.

\section{Phase 2: validation of the classification performance in differentiating between physiological and cancerous tissue by CLE examinations}

The CLE library was presented to experts $(n=3)$ independently and blinded in a random manner. They used their own expertise in CLE imaging to classify each sequence as "physiological mucosa" or "oral squamous cell carcinoma". The final ratings were statistically compared to the present gold standard for the diagnosis of oral cancer (histopathological examination). One of these three examiners evaluated all sequences for a second time 4 weeks later in order to calculate the intra-rater reliability.

\section{Phase 3: development of a classification and scoring system}

CLE experts $(\mathrm{n}=3)$ examined the footage in order to identify potential classification parameters for SCCs of the oral cavity. They were encouraged to include parameters that are simple, effective and easily applicable in daily clinical practice. The relationship between each defined parameter and the histological findings was modeled and afterwards these criteria were ranked according to their importance in identifying OSCCs. A scoring system was established on the basis of these findings in multiple discussion rounds (DOC-Score; Table 1).

\section{Phase 4: validation of the scoring system by assessing the reliability of predicting the histological classification}

All video sequences were presented to non-experts $(\mathrm{n}=3$; dentists) independently and blinded in a random manner. The inexperienced examiners made a classification based on our newly developed scoring sheet $(D O C$ Score) after being introduced to the field of CLE imaging by a unit of instruction slides. This unit presented our criteria list and explained each scoring parameter by using typical sample images of physiological tissue (Fig. 1) as well as OSCC images (Figs. 2, 3, 4) and the scoring regimen. Also potential interfering factors like artifacts or mucus at the tip of the probe were discussed in the slides. Each criterion of the questionnaire was individually based on the presence or absence of specific parameters. The sum of all single values was used to decide on a diagnosis of "physiological tissue" or "OSCC".

The ratings of non-experts were statistically compared with the results of the examinations by experts and with the histopathological assessment. One of these three non-experts assessed all sequences once again 4 weeks later in order to calculate the intra-rater reliability, as well (similar to phase 2). Inter-rater and intra-rater reliability was calculated for each of the two groups (experts and non-experts).

\section{Statistics}

Statistical analyses were conducted with SPSS version 22 (IBM, Armonk, NY, USA). We calculated occurrence frequency, significance (Chi Square) and correlation (Spearman's rho) with the histopathological result of each component of the evaluation criteria. Sensitivity, specificity and negative and positive predictive values of diagnosing cancer/non-cancer were evaluated with the help of our scoring system (non-experts) or the subjective impression (experts). Inter- and intra-rater reliability were calculated using Fleiss' kappa and Cronbach's alpha. 
Table 1 DOC-Score (diagnosing oral cancer with the help of CLE)

\begin{tabular}{|c|c|c|}
\hline Evaluation criterion & Manifestation & Score \\
\hline \multicolumn{3}{|l|}{ 1. Tissue architecture } \\
\hline \multirow[t]{3}{*}{ a) Homogeneity } & Completely organized & 0 \\
\hline & Organized + unorganized regions & 1 \\
\hline & Completely unorganized & 2 \\
\hline \multirow[t]{3}{*}{ b) Intercellular gaps } & Regular & 0 \\
\hline & Mutated & 1 \\
\hline & No longer/non-existent & 2 \\
\hline \multirow[t]{3}{*}{ 2. Cell morphology } & Consistent/regular & 0 \\
\hline & Inconsistent/dysplastic (different sizes/shapes/grey levels) & 1 \\
\hline & $\begin{array}{l}\text { Completely irregular } \\
\text { Dark, small cells } \\
\text { Blurry, cloudy image } \\
\text { Black spot (cell cluster) }\end{array}$ & 2 \\
\hline \multirow[t]{2}{*}{ 3. Fluorescence leakage } & Regular & 0 \\
\hline & Amplified (bright background) & 1 \\
\hline \multirow[t]{2}{*}{ 4. Vessels } & Regular & 0 \\
\hline & $\begin{array}{l}\text { Irregular } \\
\text { Shape (micro vessels, coiled, elongated etc.) } \\
\text { Caliber (increased) } \\
\text { Quantity (cumulated) }\end{array}$ & 1 \\
\hline \multicolumn{2}{|c|}{$x \geq 5$ points $=$ squamous cell carcinoma $(S C C)$} & Max. total: 8 points \\
\hline
\end{tabular}

CLE classification and scoring system of oral squamous cell carcinomas

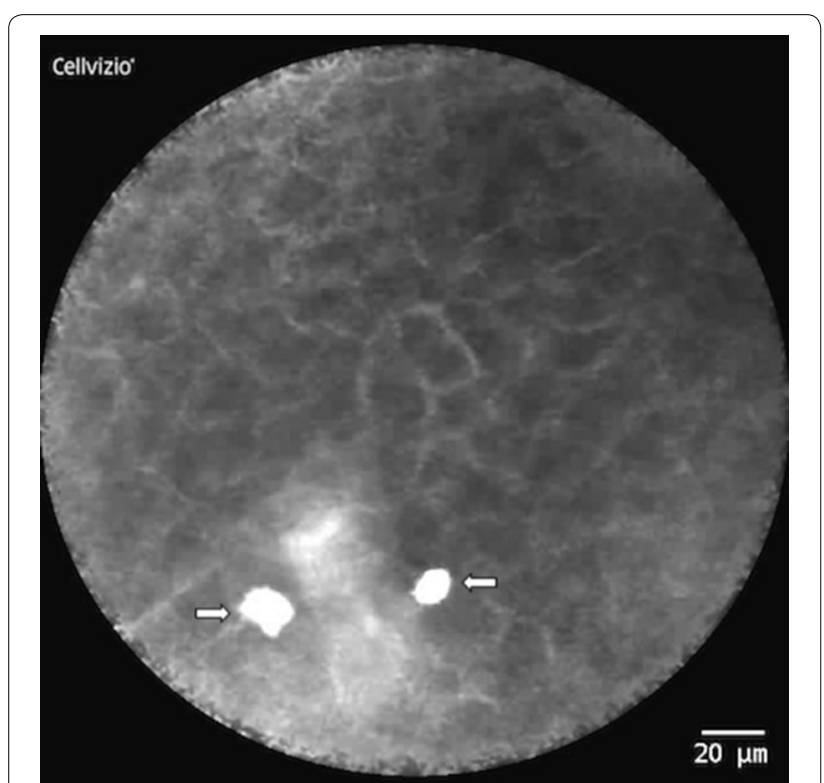

Fig. 1 CLE-image of physiological tissue (type of epithelium: attached gingiva; location: alveolar process); homogeneous visual appearance through completely organized tissue architecture (0p.) and slim, accurate intercellular gaps (0p.); consistent cell morphology in shape and color (0p.); no amplified fluorescein leakage (0p.); regular vessels (see arrows) (0p.)

\section{Results}

\section{Phase 1: CLE imaging}

A total of 95 sequences were acquired (11 sequences of the labium, 20 sequences of the alveolar ridge, 14 sequences of the palatal region and 50 sequences of cancerous tissue). The initial CLE library was reduced to representative video sequences $(\mathrm{n}=95)$ with durations between 1 and $42 \mathrm{~s}$ (total $778 \mathrm{~s}$ ) in order to remove artifacts and blind parts (physiological oral mucosa: 45 sequences/in total 260 s/median: 4 s per sequence; squamous cell carcinoma and carcinoma in situ: 50 sequences/in total $518 \mathrm{~s} /$ median: $6,5 \mathrm{~s}$ per sequence).

\section{Phase 2: validation of the classification performance in differentiating between physiological and cancerous tissue through CLE examinations}

The classification performance of the experts $(n=3)$ is shown in Table 2 (individual and averaged performance). Sensitivity and specificity in identifying OSCC as well as the positive predictive and negative predictive value for predicting a histopathological OSCC diagnosis were calculated for each expert. The averaged sensitivity of experts in diagnosing OSCCs was 0.953 , whereas specificity yielded a value of 0.889 . 

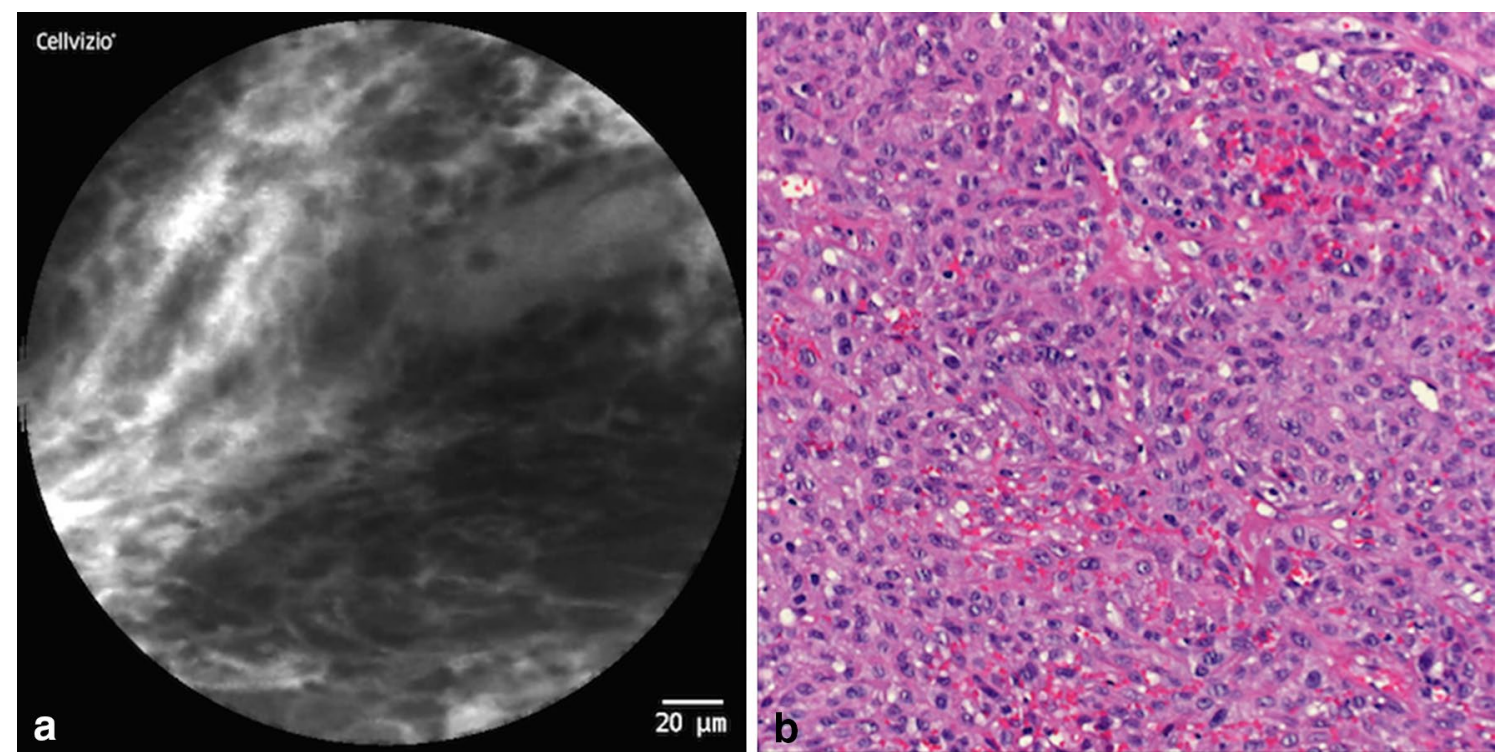

Fig. 2 OSCC (location: alveolar process); a CLE-Image: Unorganized tissue architecture (2p.); intercellular gaps not definable in some spots (2p.); irregular cells (shape, color and size) and cell cluster (=black spots; 2p.); slightly intensified fluorescein leakage (1p.); vessels not assessable (0p.); b Micrograph (HE staining) of the corresponding specimen
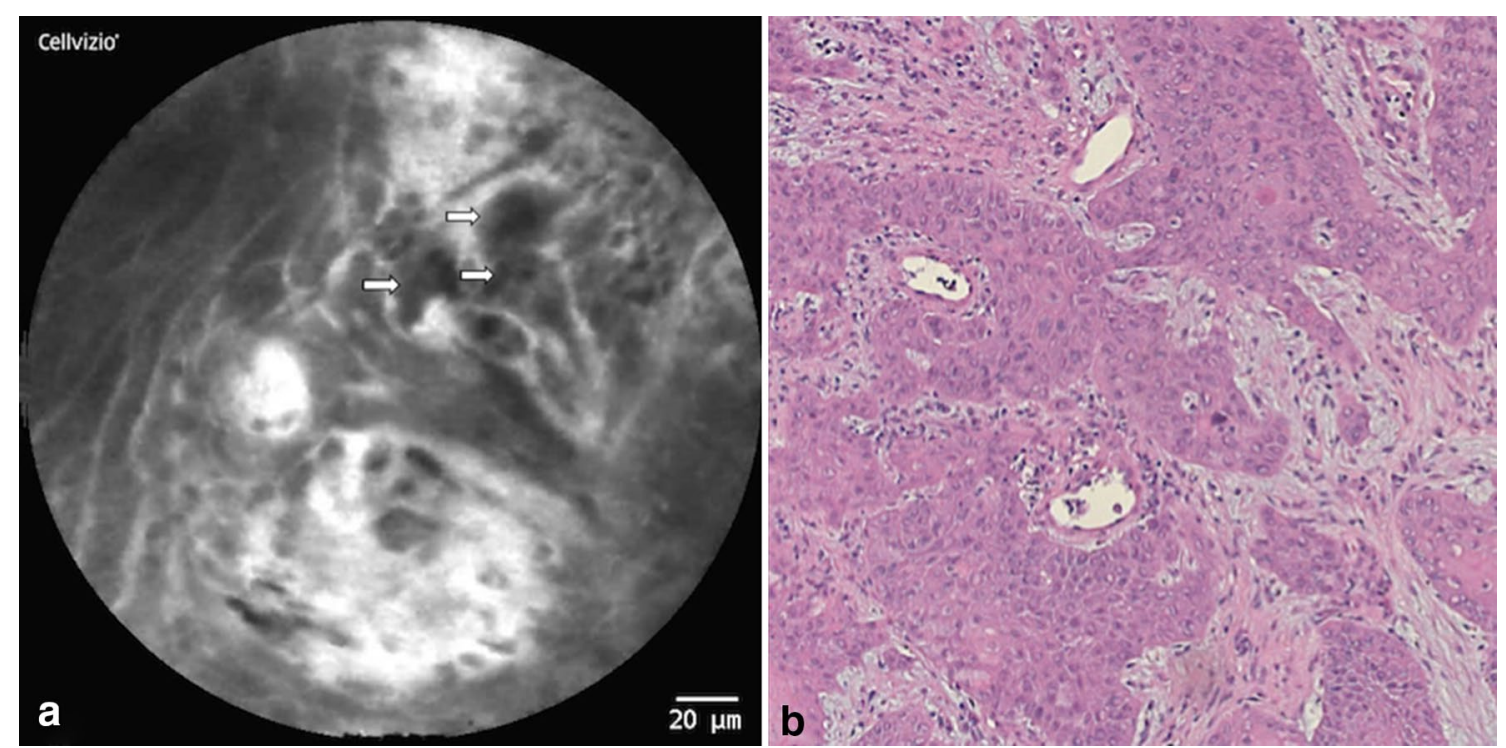

Fig. 3 OSCC (location: anterior floor of the mouth); a CLE-Image: Unorganized tissue architecture (2p.); non-existent intercellular gaps (2p.); irregular cells like cell clusters (=black spots; see arrows) (2p.); amplified fluorescein leakage (1p.); no visible vessel changes (Op.); b Micrograph (HE staining) of the corresponding specimen

The weighted Fleiss' kappa coefficient for multi-rater analysis was calculated (Table 3 ). With a value of 0.73 , substantial inter-individual agreement in diagnosing OSCCs through CLE sequences was shown. Additionally one expert subjectively rated the CLE sequences after a delay of 4 weeks. The intra-individual absolute agreement measure (Table 4) was excellent with a Cronbach's alpha of 0.989 and an intraclass-correlation coefficient of 0.979 (95\% confidence interval between 0.969 and 0.986 ). 

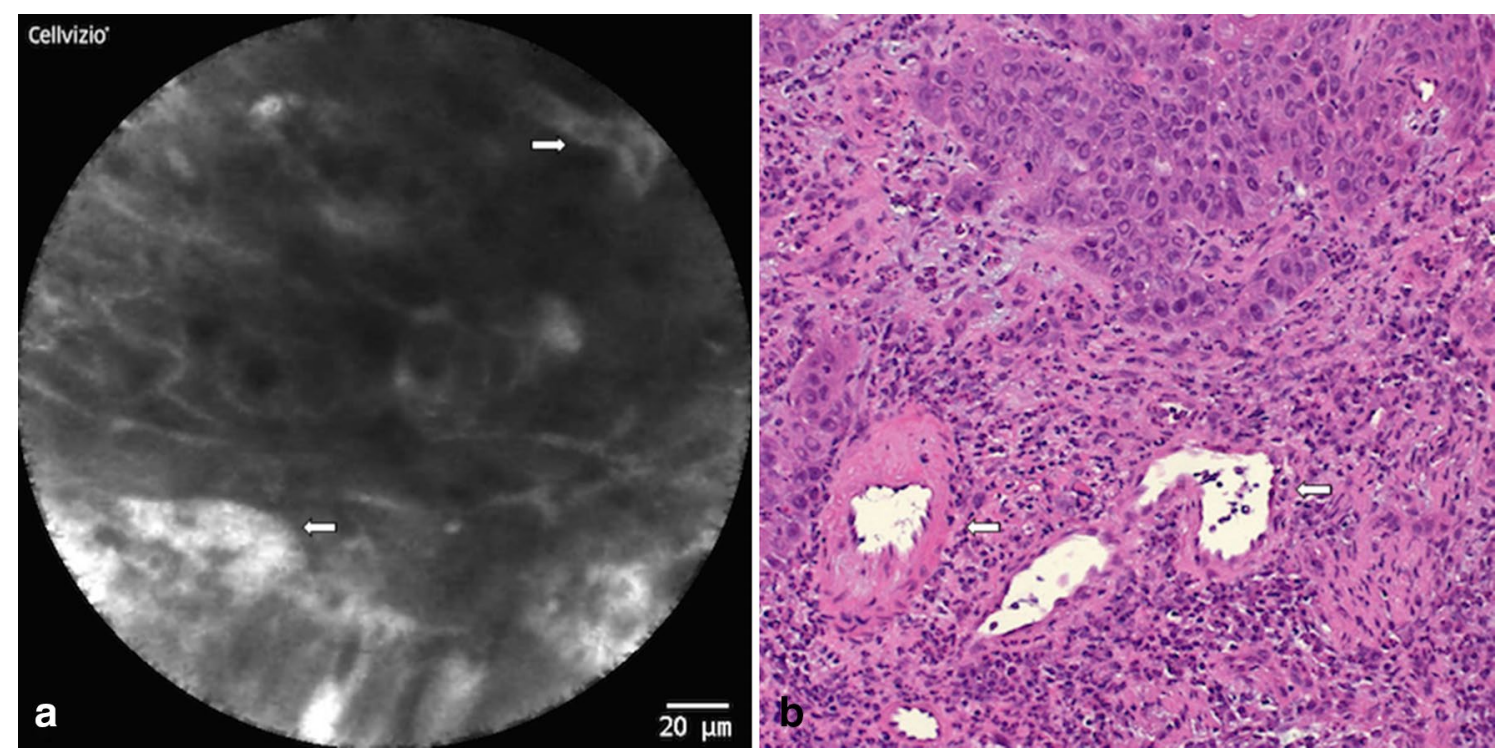

Fig. 4 OSCC (location: anterior floor of the mouth): a CLE-Image: Completely unorganized tissue architecture (2p.); non-existent intercellular gaps (2p.); blurry, cloudy image because of indistinct cell borders (2p.); no increased fluorescein leakage (0p.); irregular vessels (coiled; see arrows) (1p.); b Micrograph (HE staining) of the corresponding specimen showing similar changes in vessel morphology (see arrows), cellular structure and tissue architecture

Table 2 CLE classification performance of the experts (subjective evaluation)

\begin{tabular}{lllll}
\hline Expert & Sensitivity \% & Specificity \% & PPV \% & NPV \% \\
\hline 1 & $100(50 / 50)$ & $95.6(43 / 45)$ & $96.2(50 / 52)$ & $100(43 / 43)$ \\
2 & $86.0(43 / 50)$ & $97.8(44 / 45)$ & $97.7(43 / 44)$ & $86.3(44 / 51)$ \\
3 & $100(50 / 50)$ & $73.3(33 / 45)$ & $80.6(50 / 62)$ & $100(33 / 33)$ \\
Averaged & $95.3(143 / 150)$ & $88.9(120 / 135)$ & $90.5(143 / 158)$ & $94.5(120 / 127)$
\end{tabular}

PPV positive predictive value, NPV negative predictive value

Table 3 Inter-rater reliability

\begin{tabular}{llll}
\hline Examiner & Fleis' kappa & Agreement & \\
\cline { 3 - 4 } & & Expected & Observed \\
\hline Experts $(n=3)$ & 0.730 & 0.506 & 0.867 \\
Non-experts $(n=3)$ & 0.814 & 0.507 & 0.909 \\
\hline
\end{tabular}

Inter-rater reliability of experts (subjective evaluation) and non-experts (using DOC-score for diagnosis) in CLE assessment

Table 4 Intra-rater reliability

\begin{tabular}{lll}
\hline Examiner & $\begin{array}{l}\text { Reliability; } \\
\text { Cronbach's alpha }\end{array}$ & $\begin{array}{l}\text { Intraclass correlation; } \\
\mathbf{9} \% \text { confidence interval) }\end{array}$ \\
\hline Expert $(n=1)$ & 0.989 & $0.979(0.969-0.986)$ \\
Non-expert $(n=1)$ & 0.884 & $0.792(0.703-0.856)$ \\
\hline
\end{tabular}

Comparison of first and second assessment (video sequences; $n=95$ ) executed by one expert (subjective evaluation) and one non-expert (scoring system) with a delay of 4 weeks

\section{Phase 3: development of a classification and scoring} system

First, experts $(n=3)$ identified specific parameters that are representational of architectural and morphological tissue changes in CLE imaging. The occurrence frequency of these parameters in physiological as well as in carcinomatous CLE images is shown in Table 5. Subsequently, these items were tested according to their individual classification performance. The significance and correlation coefficients of the bivariate correlation model (score item-histo-pathological gold standard) is also shown in Table 5. Each of the score sheet items showed a significant correlation of $\mathrm{p}<0.001$ with coefficients ranging from 0.557 (fluorescein leakage) to 0.936 (homogeneity). Chi Square modeling for each item against histopathological diagnosis yielded a highly significant difference between the expected values and the observed data in each case ( $p<0.001)$. According to that, a score sheet was developed considering all important morphometric observations by experts as well as the statistical coefficient values.

During the evaluation of a CLE sequence, each criterion can be considered and rated separately with a score. Three point values $(0=$ physiological; $1=$ mildly dysplastic; 2 = highly dysplastic) were set for each of the following criteria: homogeneity, intercellular gaps and cell morphology. Fluorescein leakage and vessel morphology scores are represented by two point values $(0=$ physiological; $1=$ dysplastic). The scores are added up to a total sum that has a maximum of eight points. According to 
Table 5 Classification performance of the chosen parameters for the score sheet (by experts)

\begin{tabular}{|c|c|c|c|c|c|}
\hline \multirow{2}{*}{$\begin{array}{l}\text { Parameters } \\
\text { (score sheet) }\end{array}$} & \multicolumn{2}{|c|}{ Occurrence frequency, \%; anomalies in } & \multirow{2}{*}{$\begin{array}{l}\text { Chi square test } \\
(n=95) \\
P \text { value }\end{array}$} & \multicolumn{2}{|c|}{ Correlations $(n=95)$} \\
\hline & $\begin{array}{l}\text { Physiological mucosa } \\
(n=45)\end{array}$ & $\begin{array}{l}\text { OSCC } \\
(n=50)\end{array}$ & & $\begin{array}{l}\text { P value } \\
\text { ( } 2 \text { tailed) }\end{array}$ & Spearman's rho \\
\hline Homogeneity & $15.6(7 / 45)$ & $100(50 / 50)$ & $<0.001$ & $<0.001$ & 0.936 \\
\hline Intercellular gaps & $37.8(17 / 45)$ & $100(50 / 50)$ & $<0.001$ & $<0.001$ & 0.880 \\
\hline Cell morphology & $28.9(13 / 45)$ & $100(50 / 50)$ & $<0.001$ & $<0.001$ & 0.949 \\
\hline Fluorescein leakage & $22.2(10 / 45)$ & $78(39 / 50)$ & $<0.001$ & $<0.001$ & 0.557 \\
\hline Vessel morphology & $17.8(8 / 45)$ & $92(46 / 50)$ & $<0.001$ & $<0.001$ & 0.748 \\
\hline
\end{tabular}

Occurrence frequency of anomalies (mildly or highly dysplastic) in physiological mucosa and OSCCs, Chi square test and Spearman's correlation

Table 6 CLE classification performance of the inexperienced examiners (evaluation with newly developed score sheet)

\begin{tabular}{lllll}
\hline Non-expert & \multicolumn{2}{l}{ Sensitivity \% Specificity \% PPV \% } & \multicolumn{1}{l}{ NPV \% } \\
\hline 1 & $100(50 / 50)$ & $86.7(39 / 45)$ & $89.3(50 / 56)$ & $100(39 / 39)$ \\
2 & $98.0(49 / 50)$ & $80.0(36 / 45)$ & $84.5(49 / 58)$ & $97.3(36 / 37)$ \\
3 & $94.0(47 / 50)$ & $97.8(44 / 45)$ & $97.9(47 / 48)$ & $93.6(44 / 47)$ \\
Averaged & $97.3(146 / 150)$ & $88.1(119 / 135)$ & $90.1(146 / 162)$ & $96.7(119 / 123)$ \\
\hline
\end{tabular}

PPV positive predictive value, $N P V$ negative predictive value

the experts' opinion and their theoretic modeling of the point values for each of the items and the sum score of the sheet, a threshold of five points was identified for the differentiation between physiological and pathological CLE sequences. A point range under 5 indicates the diagnosis of "physiological tissue" (0 to 4 points), whereas a score of 5 points or higher implies the presence of an OSCC (5 to 8 points). The final score sheet is shown in Table 1 (DOC-Score).

Phase 4: validation of the scoring system by assessing the reliability of the histological classification prediction

The classification performance of non-experts $(n=3$; dentists) is depicted according to the expert ratings (Table 6). Sensitivity and specificity in diagnosing OSCCs as well as the positive predictive and negative predictive value for predicting a histopathological OSCC diagnosis were also calculated for each non-expert. The inexperienced evaluators' averaged sensitivity was 0.973 and the specificity was 0.881 . The weighted Fleiss' kappa coefficient for multi-rater analysis is also shown in Table 3. Non-experts, using the score sheet, yielded a good agreement value of 0.814 in their diagnostic performance. In order to assess the performance of the score sheet in contrast with the subjective evaluation by the experts, the intra-rater reliability (Table 4) was computed for one inexperienced examiner in a second step. The intra-individual agreement of the non-expert using the score sheet for the analysis of the CLE sequences was very good with a Cronbach's alpha of 0.884 and an intraclass correlation of 0.792 (95\% confidence interval between 0.703 and 0.856 ).

\section{Discussion}

In this study we have shown that expert differentiation between physiological and cancerous tissue in the human oral cavity with the help of chair-side CLE examination and subsequent assessment is a valid diagnostic tool with very good results compared to the histopathological evaluation.

Furthermore, we were able to ascertain that nonexperts in the field of CLE were able to make a correct diagnosis of OSCC by using the newly proposed classification and scoring system with a classification performance similar to the ratings by experts and the histopathological analysis.

The evaluation of CLE sequences is subjective and a crucial factor in making a correct assessment that requires, up to now, the experience and expertise of specialists. For that reason, and additionally as the mortality of patients critically depends on the tumor stage during the initial diagnosis [29], there is a need for a more standardized CLE evaluation for an early and accurate diagnosis of OSCC that is effective and easily applicable even for non-experts. To reach the aim of a valid and reproducible assessment in daily clinical practice, especially for examiners that are not extensively trained in the field of CLE, it was important to develop a straightforward criteria list and scoring system. To proof the validity of our newly developed scoring system (DOC-Score), we statistically analyzed non-expert ratings in the differentiation of "physiological" and "cancerous" tissue and compared it to the results achieved by experts and the histopathological assessment.

To the best of our knowledge, there is not yet a standardized procedure for the evaluation of OSCC-CLE sequences. In our study, five criteria were defined, taking 
into account the findings and expertise of our workgroup in the field of CLE. Furthermore, they were selected by correlating our findings to a number of valuable studies on tissue morphology of squamous cell cancer (SCC) in CLE imaging [3, 13, 23-26, 30].

Squamous cell tissue of the upper aero-digestive tract is known to inherit a specific vessel architecture, consisting of small intrapapillary capillary loops $[25,30]$. Deinert et al. [30] described changes in this vessel architecture for early SCCs of the esophagus via CLE imaging. Dilated and elongated intrapapillary capillary loops were reported in malignant squamous cell tissue as a fundamental parameter for tissue characterization in this study. We have found similar results in our study for SCCs of the oral cavity and thus included the vessel morphology as one main criteria in our CLE scoring system (Fig. 4). Prior studies concerning CLE imaging of SCCs focused on specific tissue patterns and cellular components that are characteristic for malignancy $[25,31]$. Liu et al. [25] identified dark epithelial cells with irregular arrangement as distinctive for SCCs of the esophagus. These characteristic changes in malignant mucosa are in accordance with OSCCs as we have found in the present study. As the study on tissue architecture as well as cell morphology is a main aspect in CLE-imaging and their alterations are found as being typical in SCCs, two evaluation criteria-namely "tissue architecture" and "cell morphology"- have been included in our scoring system. Haxel et al. investigated one patient with a carcinoma of the floor of the mouth [13] and noticed, besides irregular cell patterns and extended capillaries, a leaking of contrast agent in confocal images. Additionally Nathan et al. [23] described a disorganized epithelium with fluorescein leakage in CLE images in one case of squamous cell carcinoma of the tongue. Our findings, concerning increased fluorescence leakage in SCC, are in accordance to these findings. Therefore we integrated the fluorescein leakage as a further main criterion into our DOC-Score.

Thus, the following five parameters were defined in our DOC-Score sheet: 1. tissue architecture (1.a homogeneity, 1.b intercellular gaps), 2. cell morphology, 3. fluorescein leakage and 4. vessel morphology. This set of criteria was reviewed and adjusted in multiple discussion rounds and has been strengthened through statistical methods as well as correlation modeling to test the suitability of these items. Exemplary, histological images and their corresponding CLE images are illustrated in Figs. 2, 3 and 4.

Basically, as more dysplastic manifestations get higher scores, a high total sum indicates OSCC when a certain threshold is reached (Table 1, DOC-Score). This threshold was adjusted and validated according to expert ratings. For this purpose, video sequences not easily or clearly assessable were used by the experts to perform some exemplary calculations in order to determine the proper threshold.

Our final statistical analysis showed that the classification performance of inexperienced professionals was comparable to the subjective diagnoses by experts if the preconditions of (a) an introduction in the field of CLE imaging by standardized slide shows and (b) a rating based on our newly developed score sheet were given. Furthermore, a slightly better inter-rater agreement by laypersons was yielded when applying the score sheet. Thus, good standardization and comparability of the CLE assessment procedure can be ensured for nonexperts as well. The intra-rater reliability between the first and the second assessment of the images was better for experts than for non-experts. This suggests that, in our opinion, inexperienced evaluators need reinforcement of the learned lessons at regular intervals in order to maintain the positive learning effect and thus maintain a high level of accuracy in their diagnoses. In spite of the valuable promising results for non-experts, one has to mention that (a) data collection i.e. the handling of the CLE measuring unit and (b) the detection and selection of representative sequences is an integral part of CLE examinations. Both parts were conducted by experts in this study. The performances of the inexperienced examiners in this field of CLE examinations (data collection and sequence selection) were not subject of the present study.

In general, CLE for the imaging of bio-tissue on a microscale has yet to be applied in various medical disciplines including urology, pulmology, dermatology and gastroenterology with good prospects $[14,16,17,28]$. Recent studies showed promising results regarding a transfer of this imaging method to the area of the head and neck with a sufficient differentiation of pathological lesions to physiological tissue in the upper gastrointestinal tract like pharynx, larynx and the esophagus [3, 24-26]. Furthermore, preliminary results regarding CLE examinations in the oral cavity showed potentials for detecting cancerous lesions even in their early forms. However, these studies were mainly performed either with an experimental set-up, in ex vivo settings, on animal models or with fluorescent agents not suitable for a routine clinical investigation [3, 13, 19-22, 24-26].

A preliminary CLE study has evaluated the identification of non-dysplastic, precancerous and cancerous spots of the oral cavity by experts with high sensitivities of $80-100 \%$, as well [23]. However, no specific criteria were defined in that study prior to diagnosis regarding the diagnostic process of these entities. Furthermore, Pogorzelski et al. [3] described morphological criteria in CLE imaging of HNSCC regarding the malignant transformation of mucosal tissues. No statistical evaluation of their 
criteria has yet been performed. Both working groups used experienced investigators as raters.

Our study has some potential limitations: One of them is the number of evaluators $(n=3)$ who applied our newly developed classification and scoring system in order to validate this score. We generated significant results but there is a need for further validation of the criteria list and scoring system with a greater number of examiners (non-experts) and a larger video database. Furthermore, comparative pictures of apparently physiological tissues were generated in the same patient cohort. Consequently, we cannot completely exclude the possibility, like in similar studies of other groups [23, 32], that these visually identified physiological spots (reference points) could even be degenerated or dysplastic at an earlier stage (no biopsies with histopathological verification). Measuring points of physiological tissue were defined in advance. There is a need to generate pictures of other localizations, like the dorsal tongue (footage is difficult to generate because of the different surface composition [33]) or buccal mucosa. Prospectively, an "optical landscape" of the entire oral cavity should be created, to have the option for comparison in every part of the mouth (for example keratinized versus non-keratinized tissue). Another limitation caused by the technical conditions of pCLE is the limited penetration depth that only enables imaging of superficial mucosa. Submucosal lesion cannot be detected and monitored over time.

In this study we included patients with histopathological confirmed SCCs and differentiated between physiological and malign (OSCC) tissue with the help of the DOC-Score. In our opinion-at the current state of research-this is an essential step that should be built on further. Consequently, there is a need of research work with an enlarged patient size including other entities like precursor lesions of cancer (leukoplakia etc.) or mucosal inflammatory reactions to enable a separation of these entities towards physiological or cancerous mucosa in future.

\section{Conclusions}

In summary, CLE enables a relatively unlimited reproducibly and non-invasive "optical biopsy" for the realtime diagnosis of pathologies. In the area of oral and maxillofacial surgery, it has the potential to reduce the diagnostic time, the morbidity in the sense of an early detection as a screening method and furthermore to identify the exact location and superficial spreading of tumorous mucosa. In order to accomplish these goals, a transfer of the experimental knowledge of CLE imaging in oral and maxillofacial surgery to daily clinical practice through standardized diagnostic procedures is needed. In this study-for the first time in this specialty-with the help of pCLE, an effective and valuable scoring system was developed for the diagnosis of OSCCs. This enables a reliable classification of physiological mucosa and cancerous lesions even for non-CLE-experts in their daily clinical practice. Multicenter studies are now highly warranted to prove our initial findings.

\begin{abstract}
Authors' contributions
$\mathrm{NO}, \mathrm{FWN}, \mathrm{FS}, \mathrm{HN}, \mathrm{CK}, \mathrm{AM}$ participated in the study concept and experimental design. NO, CK, CrW have been involved in acquisition of patients and performed the recording of CLE video sequences. NO, CK, MR, HN, FS analyzed and interpreted CLE video sequences. NO, CK, WA performed the statistical analysis and interpreted the data. KB performed histological analysis of biopsies. NO drafted the manuscript. CK, HN, FS contributed to manuscript editing. MR, CVW, AM, KB, WA, FWN revised it critically. All authors read and approved the final manuscript.
\end{abstract}

\begin{abstract}
Author details
${ }^{1}$ Department of Oral and Maxillofacial Surgery, Friedrich-Alexander University Erlangen-Nürnberg (FAU), University Hospital Erlangen, Glückstraße 11, 91054 Erlangen, Germany. ${ }^{2}$ Department of Computer Science 5, FriedrichAlexander University Erlangen-Nürnberg (FAU), Martensstraße 3, 91058 Erlangen, Germany. ${ }^{3}$ Institute of Pathology, Friedrich-Alexander University Erlangen-Nürnberg (FAU), Krankenhausstr. 8-10, 91054 Erlangen, Germany. ${ }^{4}$ Department of Medical Information Technology, Biometry and Epidemiology, Friedrich-Alexander University Erlangen-Nürnberg (FAU), Waldstraße 6, 91054 Erlangen, Germany. ${ }^{5}$ Department of Medicine I, Friedrich-Alexander University Erlangen-Nürnberg (FAU), University Hospital Erlangen, Ulmenweg 18, 91054 Erlangen, Germany. ${ }^{6}$ SAOT-Erlangen Graduate School in Advanced Optical Technologies, Friedrich-Alexander University Erlangen-Nürnberg (FAU), Paul Gordan Strasse 6, 91052 Erlangen, Germany.
\end{abstract}

\section{Acknowledgements}

This work was performed in fulfillment of the requirements for obtaining the degree "Dr. med." at the Friedrich-Alexander-University Erlangen-Nürnberg (FAU). This doctoral thesis contained institutional approval from the Department of Oral and Maxillofacial Surgery and the Medical Department 1 of the Erlangen University Hospital and was conducted in affiliation with the Erlangen Graduate School in Advanced Optical Technologies (SAOT).

\section{Availability of data and materials}

At present, the acquired data material of CLE images and videos cannot yet be made publicly accessible because they are subject of further studies by our workgroup. The DOC-Score (Table 1) as newly developed tool in our study is provided in our manuscript for public use as well as to ensure an unbiased review.

\section{Competing interests}

The authors declare that they have no competing interests.

\section{Consent for publication}

Written consent from all patients included in this study was given to use the acquired data for scientific purposes including talks and publications.

\section{Ethics approval and consent to participate}

All patients were informed about potential risks and signed an informed consent form prior to the study. The study was approved by the ethics committee of the University of Erlangen-Nürnberg (reference number: 243_12 B). The study respected the principles of the ethics committee in charge as well as the 1975/1983 Helsinki declaration.

\section{Funding}

This present work was funded by ELAN-Funds, University of Erlangen-Nürnberg (reference number: 13-12-04-1-Knipfer). We acknowledge support by Deutsche Forschungsgemeinschaft and Friedrich-Alexander University Erlangen-Nürnberg (FAU) within the funding programme Open Access Publishing. The funding parties did not interfere in any way with the study design, data collection and interpretation as well as the preparation of the manuscript. 
Received: 13 January 2016 Accepted: 20 May 2016

Published online: 03 June 2016

\section{References}

1. Ferlay J, Soerjomataram I, Dikshit R, Eser S, Mathers C, Rebelo M, et al. Cancer incidence and mortality worldwide: sources, methods and major patterns in GLOBOCAN 2012. Int J Cancer. 2015;136(5):E359-86. doi:10.1002/ijc.29210.

2. Forastiere A, Koch W, Trotti A, Sidransky D. Head and neck cancer. N Engl J Med. 2001;345(26):1890-900. doi:10.1056/NEJMra001375.

3. Pogorzelski B, Hanenkamp U, Goetz M, Kiesslich R, Gosepath J. Systematic intraoperative application of confocal endomicroscopy for early detection and resection of squamous cell carcinoma of the head and neck: a preliminary report. Arch Otolaryngol. 2012;138(4):404-11. doi:10.1001/ archoto.2012.213.

4. Reibel J. Tobacco and oral diseases Update on the evidence, with recommendations. Med Principles Practice. 2003;12(Suppl 1):22-32.

5. Piazza C, Dessouky O, Peretti G, Cocco D, De Benedetto L, Nicolai P. Narrow-band imaging: a new tool for evaluation of head and neck squamous cell carcinomas. Review of the literature. Acta Otorhinolaryngol Ital. 2008;28(2):49-54

6. Kreimer AR, Clifford GM, Boyle P, Franceschi S. Human papillomavirus types in head and neck squamous cell carcinomas worldwide: a systematic review. Cancer Epidemiol Biomarkers Prev. 2005;14(2):467-75. doi:10.1158/1055-9965.epi-04-0551.

7. Neville BW, Day TA. Oral cancer and precancerous lesions. CA Cancer J Clin 2002:52(4):195-215.

8. Lingen MW, Kalmar JR, Karrison T, Speight PM. Critical evaluation of diagnostic aids for the detection of oral cancer. Oral Oncol. 2008:44(1):10-22. doi:10.1016/j.oraloncology.2007.06.011.

9. Silverman S Jr, Kerr AR, Epstein JB. Oral and pharyngeal cancer control and early detection. J Cancer Educ. 2010;25(3):279-81. doi:10.1007/ s13187-010-0045-6.

10. Paczona R, Temam S, Janot F, Marandas P, Luboinski B. Autofluorescence videoendoscopy for photodiagnosis of head and neck squamous cell carcinoma. Eur Arch Otorhinolaryngol. 2003;260(10):544-8. doi:10.1007/ s00405-003-0635-6.

11. Warnakulasuriya S, Reibel J, Bouquot J, Dabelsteen E. Oral epithelial dysplasia classification systems: predictive value, utility, weaknesses and scope for improvement. J Oral Pathol Med. 2008;37(3):127-33. doi:10.1111/j.1600-0714.2007.00584.x.

12. Karabulut A, Reibel J, Therkildsen MH, Praetorius F, Nielsen HW, Dabelsteen E. Observer variability in the histologic assessment of oral premalignant lesions. J Oral Pathol Med. 1995;24(5):198-200.

13. Haxel BR, Goetz M, Kiesslich R, Gosepath J. Confocal endomicroscopy: a novel application for imaging of oral and oropharyngeal mucosa in human. Eur Arch Otorhinolaryngol. 2010;267(3):443-8. doi:10.1007/ s00405-009-1035-3.

14. Neumann H, Kiesslich R, Wallace MB, Neurath MF. Confocal laser endomicroscopy: technical advances and clinical applications. Gastroenterology. 2010;139(2):388-92. doi:10.1053/j.gastro.2010.06.029.

15. Neumann H, Langner C, Neurath MF, Vieth M. Confocal laser endomicroscopy for diagnosis of Barrett's esophagus. Front Oncol. 2012;2:42. doi:10.3389/fonc.2012.00042.

16. Neumann H, Vieth M, Raithel M, Mudter J, Kiesslich R, Neurath MF. Confocal laser endomicroscopy for the in vivo detection of intraepithelial neoplasia in Peutz-Jeghers polyps. Endoscopy. 2010:42(Suppl 2):E139-40. doi:10.1055/s-0029-1244052.
17. Monkemuller K, Neumann H, Fry LC. Endoscopic examination of the small bowel: from standard white light to confocal endomicroscopy. Clin Gastroenterol Hepatol. 2009;7(2):e11-2. doi:10.1016/j.cgh.2008.08.047.

18. Neumann $H$, Vieth M, Siebler J, Bernatik T, Neurath MF, Boxberger F. Fluorescein-aided endomicroscopy for detection of signet ring cell carcinoma. Endoscopy. 2011;43(Suppl 2 UCTN):E199-200. doi:10.105 5/s-0030-1256380.

19. Thong PS, Olivo M, Kho KW, Zheng W, Mancer K, Harris M, et al. Laser confocal endomicroscopy as a novel technique for fluorescence diagnostic imaging of the oral cavity. J Biomed Optics. 2007;12(1):014007. doi:10.1117/1.2710193.

20. Thong PS, Tandjung SS, Movania MM, Chiew WM, Olivo M, Bhuvaneswari $\mathrm{R}$, et al. Toward real-time virtual biopsy of oral lesions using confocal laser endomicroscopy interfaced with embedded computing. J Biomed Optics. 2012;17(5):056009. doi:10.1117/1.jbo.17.5.056009.

21. Zheng W, Harris M, Kho KW, Thong PS, Hibbs A, Olivo M, et al. Confocal endomicroscopic imaging of normal and neoplastic human tongue tissue using ALA-induced-PPIX fluorescence: a preliminary study. Oncol Rep. 2004;12(2):397-401.

22. Volgger V, Conderman C, Betz CS. Confocal laser endomicroscopy in head and neck cancer: steps forward? Curr Opin Otolaryngol Head Neck Surg. 2013. doi:10.1097/MO0.0b013e32835df135.

23. Nathan CA, Kaskas NM, Ma X, Chaudhery S, Lian T, Moore-Medlin T, et al. Confocal laser endomicroscopy in the detection of head and neck precancerous lesions. Otolaryngol Head Neck Surgery. 2014;151(1):73-80. doi:10.1177/0194599814528660.

24. Chung CS, Wang HP. Screening for precancerous lesions of upper gastrointestinal tract: from the endoscopists' viewpoint. Gastroenterol Res Pract. 2013:2013:681439. doi:10.1155/2013/681439.

25. Liu H, Li YQ, Yu T, Zhao YA, Zhang JP, Zuo XL, et al. Confocal laser endomicroscopy for superficial esophageal squamous cell carcinoma. Endoscopy. 2009;41(2):99-106. doi:10.1055/s-0028-1119492.

26. Wallace M, Lauwers GY, Chen Y, Dekker E, Fockens P, Sharma P, et al. Miami classification for probe-based confocal laser endomicroscopy. Endoscopy. 2011:43(10):882-91. doi:10.1055/s-0030-1256632.

27. Reddy R, Haris M. Imaging technologies from bench to bedside. J Trans Med. 2015;13:97. doi:10.1186/s12967-015-0449-5.

28. Paull PE, Hyatt BJ, Wassef W, Fischer AH. Confocal laser endomicroscopy: a primer for pathologists. Arch Pathol Lab Med. 2011;135(10):1343-8. doi:10.5858/arpa.2010-0264-RA.

29. Coleman MP, Gatta G, Verdecchia A, Esteve J, Sant M, Storm H, et al. EUROCARE-3 summary: cancer survival in Europe at the end of the 20th century. Ann Oncol. 2003;14(Suppl 5):v128-49.

30. Deinert $K$, Kiesslich $R$, Vieth M, Neurath MF, Neuhaus H. In-vivo microvascular imaging of early squamous-cell cancer of the esophagus by confocal laser endomicroscopy. Endoscopy. 2007;39(4):366-8. doi:10.105 5/s-2007-966217.

31. Pech O, Rabenstein T, Manner H, Petrone MC, Pohl J, Vieth M, et al. Confocal laser endomicroscopy for in vivo diagnosis of early squamous cell carcinoma in the esophagus. Clin Gastroenterol Hepatol. 2008;6(1):89-94. doi:10.1016/j.cgh.2007.10.013.

32. van Staveren HJ, van Veen RL, Speelman OC, Witjes MJ, Star WM, Roodenburg JL. Classification of clinical autofluorescence spectra of oral leukoplakia using an artificial neural network: a pilot study. Oral Oncol. 2000:36(3):286-93.

33. de Veld DC, Skurichina M, Witjes MJ, Duin RP, Sterenborg DJ, Star WM, et al. Autofluorescence characteristics of healthy oral mucosa at different anatomical sites. Lasers Surg Med. 2003;32(5):367-76. doi:10.1002/ Ism.10185. 